Cahiers $d u$ MONDE RUSSE

\section{Cahiers du monde russe}

Russie - Empire russe - Union soviétique et États indépendants

$41 / 1 \mid 2000$

Varia

\title{
Références eurasiennes au Kazakhstan contemporain
}

Fabrizio Vielmini

\section{Q OpenEdition}

\section{Journals}

Édition électronique

URL : https://journals.openedition.org/monderusse/40

DOI : 10.4000/monderusse.40

ISSN : $1777-5388$

\section{Éditeur}

Éditions de l'EHESS

\section{Édition imprimée}

Date de publication : 1 janvier 2000

Pagination : 109-134

ISBN : 2-7132-1353-3

ISSN : $1252-6576$

\section{Référence électronique}

Fabrizio Vielmini, «Références eurasiennes au Kazakhstan contemporain », Cahiers du monde russe [En ligne], 41/1 | 2000, mis en ligne le 15 janvier 2007, consulté le 06 septembre 2022. URL : http:// journals.openedition.org/monderusse/40; DOI : https://doi.org/10.4000/monderusse.40 


\section{RÉFÉRENCES EURASIENNES AU KAZAKHSTAN CONTEMPORAIN}

L'IMPLOSION DE L'UNION SOVIÉTIQUE a déclenché au sein des États successeurs une quête identitaire originale, exacerbée par l'atmosphère troublée de ces dernières années qui s'exprime en partie dans de nombreuses références au terme « Eurasie », engendrant une véritable inflation sémantique de ce dernier.

Les steppes du Kazakhstan post-soviétique offrent un terrain favorable à la résurgencee d'un courant de pensée, né dans les années 20, qui a trouvé une nouvelle raison d'être dans le chaos actuel. Ce bagage théorique, transformé pour s'adapter à la situation actuelle, peut aider à répondre aux interrogations capitales qui se posent dans cette république, à savoir non seulement la fragilité du peuplement ethnique mais aussi l'incertitude relative à la position régionale d'un espace traditionnellement de transition, flottant entre différents univers culturels. En outre, dans le cadre de la réflexion suscitée par des études sur l'Asie intérieure, l'actuel eurasisme est utilisé comme un repère dans la quête de légitimation politique de la nouvelle Asie Centrale.

Cette étude a pour objet de présenter la dynamique de l'émergence de l'eurasisme en terre kazakhe. Elle emploie fréquemment, comme critère d'analyse, la civilisation, souvent maladroitement utilisée en Occident, qui est au sein du débat interne des Nouveaux États Indépendants (NEI) un outil indispensable pour la compréhension des évolutions postérieures à l'indépendance en raison de l'utilisation largement répandue qu'on a pu constater. L'étude se fonde sur une enquête menée au mois d'août 1998 au Kazakhstan¹. 


\section{Renaissance de l'eurasisme : définition et tendances}

Il faut tout d'abord se demander en quoi, à l'ère post-soviétique, consiste ce débat, gravitant autour de deux axes principaux : le premier, plutôt abstrait et culturel, soulève des questions philosophiques, le second, plus concret, essentiellement politique et géopolitique, vise les problèmes de l'éclatement de l'ancien espace de l'Union.

Le premier axe va dans le sens d'une philosophie de l'histoire héritée du premier mouvement eurasiste ${ }^{2}$ des années 20, mais qui, au sein de l'intelligentsia kazakhe, se veut une perspective historiosophique indépendante de celle de l'eurasisme classique.

Le deuxième axe, dans une perspective géopolitique, utilise l' « eurasisme » comme mode de désignation d'une nouvelle stratégie d'intégration des NEI. Ici, l'eurasisme pallie l'absence douloureuse de paradigmes sociaux et étatiques de la phase post-soviétique. Dans ce vide référentiel, les tenants de l'eurasisme s'efforcent de mettre en relief les éléments communs aux peuples de l'ancien espace de l'Union, en cherchant à dépasser des clivages de culture internes aux peuples eurasiatiques ${ }^{3}$.

Les deux axes du débat trouvent aisément un point de convergence dans l'utilisation de la philosophie de l'histoire pour justifier les appels à l'intégration et le trait d'union majeur réside dans la volonté de penser le futur de la cohabitation dans la diversité. On pourra ensuite faire la synthèse des deux niveaux de notre réflexion en appréhendant le sens contemporain de l'eurasisme comme une réaction, positive ou non, à la globalisation, comme une sorte d'idéologie post-soviétique, et enfin comme une formule exprimant une stratégie d'intégration.

Selon une autre classification, il existe des eurasismes idéologiques, politiques et même scientifiques. Sur le plan idéologique, l'eurasisme exprime une position anti-libérale, alors que, en France surtout, il a été consideré comme un courant d'extrême-droite ${ }^{4}$. L'eurasisme renaît aussi sur le plan scientifique et fait éclore une

2. Contrairement à une tendance fort répandue en France, le lecteur ne trouvera pas dans ce travail l'expression «eurasien », car nous considérons qu'il relève d'un contexte purement géographique ou de la notion de métissage. Nous lui avons préféré l'expression « eurasiste », par ailleurs beaucoup plus proche du russe evrazijstvo, pour ce qui concerne les références aux buts politiques des différents mouvements et à l'œuvre des auteurs de l'entre-deux-guerres ou de Gumilev, et « eurasiatique » pour les discours qui, hors de cette référence, cherchent les fondements de la cohabitation entre peuples post-soviétiques.

3. A. C. Panarin, « Meždu neprimirimoj vraždoj i nerazdel’́nym edinstvom » (Entre une haine inextinguible et une unité indivisible), Voprosy filosofii, 6, 1995, pp. 29-55, actes d'une table ronde.

4. Ainsi on a parlé d'un néo-eurasisme, sorte de fascisme russe de fin de siècle (voir Françoise Thom, «Eurasisme et néo-eurasisme », Commentaire, été 1994, pp. 303-309). Cette conclusion, liée à la grande visibilité médiatique des franges nationalistes russes qui se définissaient comme la "nouvelle droite russe » et qui manipulaient la doctrine, ne tient pas. Ceci, en premier lieu parce qu'il y a dans le parti communiste de Zjuganov l'idée qu'une civilisation eurasiatique a remplacé la lutte de classes en la reléguant au rang de « superstructure » d'une lutte qui a lieu entre philo-occidentaux et eurasistes (G. Zjuganov, « La Russia al bivio », Il calendario del popolo, giugno 1996, p. 599). Il faut savoir qu'on se trouve ici au centre d'un problème général du débat politique russe et post-soviétique contemporain, où les catégories droite/gauche n'expliquent plus grand-chose. Surtout, la conclusion de Françoise Thom ne résiste pas à la lumière de la longue durée braudélienne dans laquelle s'insère la réflexion eurasiste. 
pléthore de travaux parfois contestables, parfois dotés d'éléments considérés comme scientifiques. De nombreux savants au Kazakhstan se réclament de l'héritage de Lev Nikolaevič Gumilev. En effet, l'œuvre de cet historien et anthropologue dont l'ambition fut d'octroyer aux peuples de la steppe une identité culturelle, a été, pendant le vide de références explicites à l'eurasisme de la période soviétique, le principal trait d'union entre la réflexion des années 20 et la réflexion contemporaine ${ }^{5}$.

Même si ces deux antécédents intellectuels ne définissent qu'une petite partie de la question (surtout au Kazakhstan), il faut néanmoins toujours garder à l'esprit leurs formulations en tant que principaux points de repère pour s'orienter dans l'actuelle inflation et l'omniprésence du terme « Eurasie ».

\section{Les acteurs du discours eurasiste}

On peut considérer comme "pôles » toutes les réalités sociales où, en deçà du discours structuré des centres intellectuel, politique ou scientifique, la référence à l'Eurasie offre des nuances. Nous définissons les pôles selon plusieurs paramètres, en termes ethniques par exemple; en effet on peut relever des nuances très différentes si l'on compare la perception de l'eurasisme par les Russes ou par les Kazakhs. La référence ethnique est doublée d'une localisation dans l'espace, selon que l'on distingue les Kazakhs en Russie, les Kazakhs du nord de la république, ou leurs compatriotes du sud. Cette différenciation peut aussi se faire sur une base ethno-tribale. À cela s'ajoutent encore les clivages religieux, étant donné que les mêmes hiérarchies musulmanes et orthodoxes, entretenues au cours des générations par les systèmes impériaux de Moscou, sont intervenues plusieurs fois, et dans un sens positif, dans la course à la définition de l'Eurasie ${ }^{6}$.

Les pôles et leur action sont fortement tributaires de leur relation avec le pouvoir officiel. Dans certains cas, ils constituent des centres d'opposition, dans d'autres ils sont soigneusement entretenus par le gouvernement, auquel cas ils ne sont souvent rien d'autres que de pseudo-groupes scientifiques adoptant une ligne favorable au pouvoir. Au contraire, la quête de l'Eurasie dans les groupes de l'opposition peut

5. Même si on a pu voir des effets de cette perspective dans l'action de Hruščev - théorisation sur la formation imminente d'un homo sovieticus à travers une politique de rapprochement et de synthèse des peuples soviétiques nommée sbliženie (rapprochement), qui appartint également à la doctrine officielle de Brežnev.

6. Malheureusement, on n'a pas réussi à interroger les acteurs religieux kazakhs. On suppose opérationnel le modèle observé du côté russe où les religieux orthodoxes et musulmans tiennent un discours fort semblable. Ils parlent, en effet, de « temps des troubles » et se rapprochent de la dénonciation eurasiste de l'Occident, qui a intérêt à la décomposition future de l'espace postsoviétique. Voir les deux entretiens, d'une part avec le métropolite de Smolensk et de Kaliningrad, Kiril, et d'autre part avec le mufti de la Russie centre-européenne, Ravil Gainutdin, respectivement parues sous le titre : «Sohranim svoju samobytnost' i duhovnost' » (Conservons notre originalité et notre spiritualité), et « Mir spaset dobrota » (La bonté sauve le monde), Evrazija, narody, kul'tury, religii, III, 1, 1995, pp. 18-23 et pp. 23-26. 
parfois être dangereuse pour les chercheurs engagés, à en juger par les risques encourus comme ce fut le cas récemment en Azerbaidjan ${ }^{7}$.

Les différents discours, constamment reformulés, sont donc utilisés et instrumentalisés selon le pôle dont ils émanent, mais plus encore selon les récepteurs à l'intention desquels ils sont élaborés. L'individualisation des destinataires distingue un clivage général intérieur/extérieur. Les « cibles » peuvent être la population, qu'on veut soustraire aux craintes de conflits inter-ethniques ou projeter audelà des misères du quotidien, mais aussi les partenaires de la CEI, auprès de laquelle on tente de se présenter comme un allié solidaire, ou encore d'autres acteurs internationaux.

La pluralité des messages et des acteurs est compliquée par les distorsions qu'ils subissent de la part des diffuseurs médiatiques et humains de leur expression. Les lignes de la redéfinition identitaire des NEI sont en fait massivement influencées par la géopolitique régionale et les tendances culturelles perçues à travers les médias internationaux, ce qui renforce souvent les contradictions évidentes entre les différentes interprétations de l'Eurasie.

\section{Enjeu de civilisation dans l'eurasisme}

L'enjeu le plus intéressant du nouvel eurasisme consiste à redéfinir les bases des sociétés post-soviétiques. Ces dernières années, on a vu, partout dans les NEI, les nationalismes intransigeants de la première heure laisser la place à de nouvelles formes d'interrogations identitaires où la notion de « civilisation » a son importance, en tant qu'aire culturelle dépassant les frontières des États et des nations, fondée sur l'idée d'un développement historique commun. Pour la science européenne, il s'agit de repenser cette notion au-delà des schématisations simplistes et conflictuelles de Samuel Huntington ${ }^{8}$; ainsi cette catégorie était déjà au cœur de la défense du pluralisme culturel de l'eurasisme des années 20, qui appréhendait les autres civilisations comme autant de miroirs pour la compréhension de soi-même. De nos jours, l'eurasisme propose un cadre philosophique propice à une interrogation sur les rapports entre les cultures au-delà de modèles uniformisants occidentaux mais impropre à une utilisation en tant que catégorie sociologique 9 .

En fait, comment établir les limites d'une civilisation qui, outre qu'elle occupe un espace, est une combinaison de normes de comportements et de stéréotypes stables définissant les relations sociales? Dans le contexte eurasiatique, il ne faut pas sous-estimer le degré de structuration de la société héritée du système soviétique. Cette phase historique, d'une ampleur unique dans l'histoire de l'humanité, est considérée par les analystes même les plus modérés comme un moment poten-

7. Moskovskie novosti, 3/5/1998.

8. S. Huntington, Le choc des civilisations, Paris, Odile Jacob, 1997.

9. I. N Sizemskaja, « Političeskaja doktrina ili istoriosofskaja koncepcija » (Doctrine politique ou conception historiosophique », in A. C. Panarin, « Meždu neprimirimoj vraždoj... », art. cit. 
tiel de création culturelle, qui ne put aboutir par manque de temps. Ainsi, selon l'orientaliste G. V. Miloslavskij, à l'intérieur de l'URSS, « un système civilisationnel intégral » était en train de se constituer. D'autres chercheurs voient dans le système soviétique, sinon une civilisation, du moins un «substitut des références universelles qui composent une civilisation avec ses structures spirituelles et sociales $»^{10}$. Dans ce cadre, l'Asie Centrale, dont la société restait en même temps largement réglée à plusieurs niveaux par des principes musulmans, venait «à se situer dans deux dimensions $»^{11}$.

Selon cette optique, le Kazakhstan se présente comme la juxtaposition d'au moins trois ensembles culturels/civilisationnels. Tel est le discours eurasiste des acteurs kazakhs d'aujourd'hui. Ainsi, les auteurs du projet d'Union eurasiatique, Askar Ahmedzanov et Alma Sultangaleva, ont justifié l'orientation de la diplomatie du pays en décrivant le processus d'ethno-polarisation qui le place dans la sphère d'attraction des deux centres distincts incarnés par la Russie et l'Asie Centrale ${ }^{12}$, se manifestant sur les plans économique, culturel et social. Pour le président Nazarbaev, l'essence de la transition réside dans la fondation de réalités historiques neuves qui modifient en profondeur la structure des interactions étatiques ${ }^{13}$. L'émergence de nouveaux intérêts économiques souvent contradictoires conduit à une « différenciation significative du paysage politique (političeskij landšaft), à un schisme croissant dans les valeurs culturelles et plus largement dans les orientations civilisationnelles (civilizacionnye orientacii) ${ }^{14}$.

\section{Russie-Kazakhstan-Europe : attraction et différenciation}

La question se pose autant pour le Kazakhstan que pour la Russie. Les incertitudes du Kazakhstan proviennent du caractère de la présence russe en Asie Centrale, qui franchit les limites géostratégiques de ce théâtre pour atteindre une dimension d'ordre métapolitique et culturel ${ }^{15}$. En comparant les relations étroites qu'elle entretient avec le Kazakhstan et la volonté de divorce affichée par les Ukrainiens, la Russie a pu mesurer l'absurdité politique du panslavisme pour un pays qui doit

10. B. Erasov, « Rossija v central’noaziatskom geokul’turnom komplekse » (La Russie dans le complexe géo-culturel centrasiatique), Rossija i musul'manskij mir, 1, 1994, p. 6.

11. G. V. Miloslavskij, «Integracionnyj potencial regiona » (Le potentiel d'intégration de la région), Vostok, 5, 1996, p. 8.

12. M. M. Tazin, ed., Evrazijskoe prostranstvo : integracionnyj potencial i ego realizacija (L'espace eurasiatique : potentiel d'intégration et réalisation), Almaty, Kazahstan, 1994.

13. N. A. Nazarbaev, «Evrazijskij sojuz: novye rubeži, problemy, perspektivy » (L'union eurasiatique : nouvelles frontières, problèmes, perspectives), in Pjat' let nezavizimosti (Cinq ans d'indépendance), Almaty, Kazakstan, 1996, pp. 430-442.

14. Ibid., p. 432.

15. A. Kortunov, A. Ÿumihin, «Russian policies toward Central Asia: An overview », in R. Z. Sagdeev, S. Eisenhower, eds, Central Asia : Conflict, resolution and change, New York, The Center for Post-Soviet Studies, 1995. 
impérativement trouver une forme institutionnelle apte à gérer une société hybride turco-slave. La Russie doit choisir entre un modèle de développement européen ou asiatique. Cette confrontation, en touchant la véritable raison d'être de la Russie en tant qu'État et société, révèle une très profonde dichotomie amour-haine, issue de la dialectique millénaire entre monde nomade et monde sédentaire ${ }^{16}$. Les séquelles indélébiles du «joug mongol » et de ce qui constitue le «barbare » ${ }^{17}$ ne peuvent laisser indifférent le peuple kazakh dont les instruments intellectuels pour la compréhension du monde se sont formés à travers la pensée russe. Cette transition culturelle, de moins d'un siècle, a projeté dans les steppes l'acquis le plus avancé des idées européennes. Les Kazakhs ont subi, dans des termes liés à la fois à l'idéologie officielle et à leur dépendance intellectuelle historique vis-à-vis des Russes, une marginalisation de leur ethnicité censée être « inférieure », barbare, par rapport à l'Europe et aux Empires sédentaires, ce qui permettait aux Russes de compenser par leur «mission civilisatrice» en Asie leur sentiment d'infériorité face aux Européens.

Depuis 1991, on assiste à un renversement des définitions soviétiques de la nationalité, de la citoyenneté, de l'État, qui ponctuent le débat sur l'identité postsoviétique. Donc, comme dans le cas du premier eurasisme russe, le Kazakh lui aussi assume dans son discours le poids du passé asiatique en tirant sa force d'une redécouverte de l'Asie, qui rééquilibre la dignité réciproque des deux peuples. Cette reconnaissance du passé nomade, divergente de la conception longtemps dominante en Russie - et bien entendu en Occident - , pose la question eurasiste au Kazakhstan; cette question a été ouverte, il y a vingt-quatre ans, avec la publication de Az i Ja par Oljas Sulejmenov ${ }^{18}$. Dans ce texte, publié en 1975 et semicensuré jusqu'en 1991, Sulejmenov examine les bases de l'epos russe et met en évidence des liens de langue et de sang qui mettent en question les visions traditionnelles de la civilisation russe étrangère au monde nomade.

La référence eurasiste synthétise aussi les attitudes envers l'Europe. Il est vrai, comme il a été dit, que la référence eurasienne au Kazakhstan révèle une volonté de se projeter vers l'Europe. Mais il est vrai aussi que la réalité politique d'une société figée dans un rapport structurellement paternaliste avec le pouvoir - confirmé par l'évolution politique actuelle à la fois à Moscou et à Astana - exclut l'adoption intégrale des conceptions politiques européennes. Il faut donc assumer (et, si possible, avec orgueil) cette réalité, au lieu d'en avoir honte devant l'Occident, en utilisant l'héritage historique du premier eurasisme qui défendait le pluralisme

16. Ainsi on en est revenu à entendre des déclarations du genre «L'Asie compromet moralement la Russie », résurgence évidente du thème du « péril jaune ». Voir J. Latynina, « L'idea russa e il nuovo sistema statale della Russia: problemi, orientamenti, prospettive », in P. Sinatti, Che cosa vogliono i russi, Milan, Theoria, 1993, p. 76, traduction italienne d'une table ronde de la Fondation Gorbačev, publiée dans Novyj mir, 1, 1993.

17. H. Eitzen, «Refiguring ethnicity through Kazak genealogies », Nationalities Papers, XXVI, 3, 1998,pp. 433-451.

18. O. Sulejmenov, Az i Ja, Kniga blagonamerennogo čitatelja (Az i Ja [A et Z], le livre d'un lecteur bien intentionné), Alma-Ata, Zazušy, 1975. 
culturel et dénonçait l'uniformisation imposée par l'occidentalisation ${ }^{19}$. La présence massive des intérêts étrangers, souvent dans des termes très proches de l'exploitation coloniale, rappelle la réaction anti-occidentale des années 2020, quand l'Occident fut dénoncé comme porteur de valeurs incompatibles avec ces peuples qui ne connaissaient pas de voie intermédiaire entre État fort et anarchie ${ }^{21}$. L'élément, considéré comme «barbare » aux yeux des Européens, est considéré au contraire par les Eurasistes comme une ligne de force de cette souche culturelle.

\section{Kazakhstan-Orient-Ummah : la dérive}

Les suggestions venant de l'Occident et du Nord ne sont pas les seules causes du rayonnement de l'eurasisme dans la société kazakhe. Dans une perspective braudélienne à long terme, les rapports du pays avec les voisins centrasiatiques et orientaux peuvent aussi être l'objet d'interrogation 22 .

$\mathrm{Si}$ on fait abstraction de l'histoire soviétique récente, on voit bien comment le Kazakhstan demeure dans un processus géopolitique ${ }^{23}$ ouvert par l'afflux des civilisations bouddhistes et confucéennes de l'Est ${ }^{24}$. Comme en témoignent toutes les épopées, c'est la «grande retraite»(1723-1728) engendrée par le retour de la Chine dans la région, et non pas l'arrivée des Russes, qui demeure le fait central dans la formation de l'image collective de l'Ennemi. Aujourd'hui, les massacres périodiques perpétrés par Pékin sur les peuples turciques du Xinjiang, dont les Kazakhs sont la deuxième formation, exacerbent une appréhension séculaire décuplée par la nouvelle puissance mondiale du voisin.

Cependant il existe un clivage important entre les Kazakhs, peuple du monde musulman ex-soviétique le plus éloigné de la rigidité de l'Islam, et leurs voisins méridionaux. Ce décalage, presque toujours sous-estimé par des analystes anxieux d'opposer le sud musulman au nord orthodoxe, ne relève pas seulement du caractère folklorique des préceptes coraniques adaptés au chamanisme de la steppe ${ }^{25}$, mais aussi des déclarations de certains nationaux kazakhs se targuant d'être une nation turcique plus «pure » que la nation ouzbeke, influencée par les populations urbaines du sud ${ }^{26}$, ce qui marquerait la frontière avec les Ouzbeks ${ }^{27}$. Parmi ces

19. E. Bagramov, «Rossija i proekt Evrazijskovo Sojuza (La Russie et le projet d'Union eurasiatique), Evrazija, narody, kul'tury, religii, III, 1, 1995, pp. 18-23.

20. N. Trubeckoj, «Russkaja problema » (Le problème russe), Na putjah, Berlin, 1922, pp. 294-316.

21. Entretien avec Azdar Aširovič Kurtov de l'Institut russe d'études stratégiques de Moscou (RISS), Almaty, 7/8/1998.

22. Tous nos entretiens avec des personnalités de la vie publique kazakhe ont soulevé ce thème.

23. Pour le concept de processus géopolitique, cf. Pascal Lorot, François Thual, La géopolitique, Paris, Montchrestien, 1997, p. 75.

24. René Grousset, L'Empire des steppes, Paris, Payot, rééd. 1997, p. 587.

25. K. H. Karpat, «The roots of Kazakh nationalism: Ethnicity, Islam or land?», in M. Buttino, ed., In a collapsing Empire, Milan, Feltrinelli, 1993, p. 313. 
derniers, l'envie de se redéfinir comme une nation en harmonie avec leur communauté confessionnelle et culturelle a engendré des changements dans le profil culturel du quotidien ${ }^{28}$. Tandis que le choix du changement d'alphabet exprime la volonté des Ouzbeks d'orienter l'ensemble de la vie nationale vers une aire culturelle précise, des moments forts de vécu commun, comme la Grande Guerre patriotique, s'effacent progressivement alors que les représentations de l'histoire récente, où les éléments de coercition du rapport avec les Russes sont exacerbés, sont plus présentes et que le facteur musulman se fait plus marquant. Finalement, la faiblesse des liens d'intégration régionale centrasiatique correspond à une volonté précise des Kazakhs de se démarquer d'un voisin inconfortable et d'une identité centrasiatique ressentie par la majorité des couches dirigeantes comme pénalisante pour un pays qui se veut un pont entre l'Europe et l'Asie et, dans un certain sens, un creuset formé par la rencontre d'apports culturels différents.

\section{Aspect géopolitique de l'Eurasie au Kazakhstan}

Comme une civilisation peut se définir tout d'abord à travers son théâtre, le milieu géographique où se déroulent les modifications politiques et économiques qui sont interprétées comme changement civilisationnel ${ }^{29}$, la question kazakhe peut être vue aussi sous l'angle concret de problèmes spatiaux.

Faute de définition convenant à l'ensemble géopolitique longtemps homogène que représentait l'URSS, l'Occident s'est rabattu sur la dénomination d'Eurasie. Terme neutre, l'Eurasie relève plutôt dans les NEI d'une construction géopolitique en compétition avec d'autres. Ainsi, conformément à la définition d'Yves Lacoste $^{30}$, il est possible d'envisager l'Eurasie comme une représentation géopolitique résultant de la concurrence avec d'autres espaces intéressant le Kazakhstan.

En premier lieu la Turquie a, elle aussi, élaboré une conception de l'Eurasie en raison de sa position géostratégique, se trouvant ainsi en concurrence avec la Russie dans son rôle de pont entre l'Europe et l'Asie Centrale. Au Kazakhstan, Ankara a

26. Entretien avec Imankul Nurtas, responsable de la chaire de philosophie de l'Université du Kazakhstan du sud, Ÿymkent, 3/9/1998.

27. Aitkazin Teliszan, responsable de la chaire de philosophie de l'Université eurasiatique «Lev Gumilev» d'Astana, 28/8/1998. M. Teliszan a aussi affirmé que ce clivage « civilisationnel » porte également sur le manque d'enracinement de l'esprit commercial dans les mœurs populaires des Kazakhs, qui en viennent à mépriser les targošniki ouzbeks.

28. Stéphane Dudoignon, « Changements politiques et historiographiques en Asie Centrale », Cahiers d'Études sur la Méditerranée orientale et le Monde turco-iranien, 16, 1993, p. 117.

29. Fernand Braudel, Grammaire des civilisations, Paris, Flammarion, 1993.

30. On se réfère à l'œuvre de ce géographe pour souligner le fait que les renvois à l'Eurasie sont à la fois des auto-perceptions (des images du réel construites à travers l'expérience) et des représentations symboliques et théâtrales reliées par une mémoire sélective aux longues durées historiques et proposées avec des objectifs de mobilisation dans un milieu conflictuel et dramatique comme l'espace post-soviétique, Yves Lacoste, ed., Dictionnaire de géopolitique, Paris, Flammarion, 1993, préambule, p. 4. 
évité d'utiliser le terme d' «Eurasie » qui correspondait à une doctrine officielle du gouvernement ${ }^{31}$.

À présent en Russie, mais surtout en Asie Centrale, la géopolitique est l'objet d'un très grand intérêt comme le prouve l'existence d'une multitude de centres d'analyse stratégique, où la réflexion spatiale est dominante, et qui sont devenus grands producteurs de représentations géopolitiques. Le Kazakhstan est sûrement le NEI où la transition a fait surgir l'appareil de politique extérieure « indigène » le plus actif exprimant des représentations neuves et ambitieuses, dont en premier lieu l'eurasisme, avec pour objectif la valorisation de la position de la république ${ }^{32}$. Celle-ci est conçue par les géopoliticiens locaux comme le pivot des relations internationales entre le Pacifique et l'Europe. Un tel esprit est évident si l'on considère le projet d'Union eurasiatique (Evrazijskij Sojuz Gosudarstv), ou les initiatives visant à bâtir un pendant asiatique de l'OSCE, ou encore les engagements dans les rapports avec la Chine. On a pu constater un intérêt extrême pour les conceptions de la géopolitique classique allemande et pour la pensée de Samuel Huntington ${ }^{33}$.

\section{Le Kazakhstan est-il en Asie Centrale?}

Ces spéculations peuvent se développer dans la mesure où l'Asie Centrale est une notion artificielle, une appellation forgée par les savants européens, à la fin du $\mathrm{XIX}^{\mathrm{e}}$ siècle, pour désigner un espace, resté en dehors des grands empires voisins, connu jadis seulement pour son instabilité et son hétérogénéité34 ${ }^{2}$ Les Kazakhs se trouvaient placés devant différentes options - chinoise, tsariste, turkestanaise dont on remarquera que seule l'une d'entre elles relève du monde centrasiatique ${ }^{35}$. En 1991, les pouvoirs post-communistes de cette région ont voulu supprimer la subdivision soviétique, et ont déclaré par un acte symbolique que le Kazakhstan faisait partie de l'«Asie Centrale» (Central'naja Azija). Cet acte médiatique accompli, les dirigeants kazakhs ont vu se transformer cet espace, dépourvu d'unité géo-économique pour ses échanges, éclaté entre le nord, le nord-est et le sud.

31. Au-delà des possibilités de ces stratégies, il ne semble pas qu'on puisse parler d'une Eurasie turque. D'abord la conception, pour autant qu'elle reste floue, se fixe dans le sens géographique d'espace centripète sur la continentalité. Ensuite la vision eurasiste, en tant qu'interrogation identitaire directement liée à l'hétérogénéité culturelle et ethnique, exclut toute référence mono-ethnique, ce qui serait le cas sous la houlette d'Ankara. Si la Turquie est géographiquement « eurasiatique », elle peut à la limite utiliser cette notion dans ses rapports avec l'Europe occidentale. Pour l'espace centrasiatique, elle doit plutôt se référer à un « grand Turkestan ».

32. Notamment l'Institut d'études stratégiques d'Almaty (KISS), éditeur de la revue Kazahstan i mirovoe obščestvo, devenue Kazahstan spektr où le point de vue géopolitique est largement dominant.

33. Entretien avec Konstantin L'vovič Syroeznik, directeur adjoint du KISS, 7/8/1998.

34. Vincent Fourniau, « La transition depuis 1992 : politique et institutions des États », in Les pays de la CEI, Paris, La Documentation française, 1997.

35. S. Kljaštornyj, «Geopolitičeskaja perspektiva i civilizacionnyj vybor » (Perspective géopolitique et choix de civilisation), Nezavisimaja gazeta, 24/2/1996. 
Chaque État y évolue selon des modèles de développement différents en s'éloignant de ses voisins, ce qui l'amène à s'interroger sur sa propre position par rapport au monde extérieur.

La fin de la division bipolaire du monde a réactivé les potentialités d'un autre espace qui était resté longtemps en dehors du jeu des relations internationales. Il s'agit d'un «Croissant islamique » comprenant des territoires qui correspondent aux peuples d'origine turcique et persane, tampon entre la civilisation arabe et le monde slave oriental, auxquels il va se juxtaposer dans plusieurs secteurs. Après des siècles de relations internationales caractérisés par des formations impériales stables, l'avancée des Talibans et les troubles islamistes au Tadjikistan et dans le Fergana, ont fait penser enfin à une réorientation géopolitique régionale causée par l'intrusion de l'Afghanistan, de l'Iran et du Pakistan ${ }^{36}$. Les réactions en Asie Centrale devant cette réémergence sont fort différentes. Tout en étant menacés eux aussi, les Ouzbeks subissent la tentation d'entrer dans un ensemble où ils pourront jouer le rôle de moyenne puissance, rôle impossible si l'intégration au Nord subsiste. Les Kazakhs au contraire sont très préoccupés par la tournure des événements, d'autant plus que leurs régions méridionales, en raison des catastrophes socio-économiques de la phase post-communiste, subissent l'attraction du Croissant. La question est posée de savoir où s'établira la frontière de cette confrontation, au sud ou au nord de la frontière du Kazakhstan et de la Kirghizie?

Au-delà du monde musulman, la position incertaine du Kazakhstan alimente un syndrome, où l'Occident, les grands desseins panturquistes, ou encore les forces jaillissantes des peuples de l'Extrême-Orient semblent investir lentement les régions du grand continent affaiblies par la crise. Après les événements de Tchétchénie, beaucoup ont pressenti l'éventualité d'un déclenchement d'une gigantesque revanche ethnique. C'est dans cette optique que V. Žirinovskij, ainsi que le chef du PC russe, G. Zjuganov, se sont intéressés à la position du Kazakhstan. Zjuganov en particulier est convaincu de l'existence d'une manœuvre visant à mettre les héritiers de l'URSS dans un « arc de la crise qui, du Tadjikistan à travers le Caucase du nord, la Crimée et le Dniestr gagne les républiques baltes ${ }^{37}$. L'actuel eurasisme reprend l'analyse géopolitique classique de l'antagonisme irréductible entre terre et mer $^{38}$, qui continue à opposer l'espace post-soviétique au monde à direction anglo-saxonne. La référence eurasiste apparaît au Kazakhstan comme l'outil polémique d'une opposition que l'on retrouve dans les affirmations des membres du Congrès du peuple du Kazakhstan :

36. Pour certains chercheurs, la « région centrasiatique » est un prisme d'observation privilégié pour la compréhension des dynamiques post-bipolaires. V. Belokrinickij, « Genezis i osnovnye harakteristiki regiona » (Genèse et caractéristiques fondamentales de la région), in Central'naja Azija. Puti integracii v mirovoe obščestvo (L'Asie Centrale : les voies d'intégration dans la société mondiale), Moscou, 1995, pp. 9-38.

37. Il faut noter que Zjuganov n'a pas explicité qui se trouverait derrière cette stratégie. M. M. Tazin, ed., Evrazijskoe prostranstvo..., op. cit.

38. D. Kerr, « The new Eurasism : The rise of geopolitics in Russia's foreign policy », EuropeAsia Studies, 47, 6, 1995,pp. 977-988. 
«Ils ont été les champions du 'nouvel ordre mondial' qui ont profité de l'exigence urgente des réformes pour perpétrer une destruction totale, voler la propriété nationale du peuple et tenter de convertir un État puissant en un groupe démembré de territoires coloniaux dépendants. Quand ils ont déchiré le pays, ils ont égorgé un organisme vivant. »39

Ces affirmations aident à comprendre comment de nombreuses références à une Eurasie comme espace de stabilité proviennent justement des craintes suscitées par la perspective de $\ll$ Balkans eurasiatiques $»^{40}$.

Plus généralement il est utile de relier le rayonnement de la proposition eurasiste aux répercussions sur la société d'une évolution politique qui a entraîné le pays dans un schéma colonial type, où une classe restreinte de bourgeoisie compradore, seule bénéficiaire de l'ouverture des frontières, draine les capitaux du pays hors de ses frontières ${ }^{41}$. Cela, combiné à la perte générale d'enthousiasme pour l'Occident, donne du crédit aux dénonciations eurasistes de l'impérialisme occidental et au discours sur l'organisation géoéconomique du continent.

\section{Les intellectuels kazakhs et la nécessité d'une redéfinition identitaire}

Le discours eurasiste demeurant un phénomène intellectuel, il semble indispensable d'étudier cette couche sociale qu'est l'intelligentsia kazakhe, aisée et respectée sous le pouvoir soviétique (et déjà même sous le pouvoir tsariste), mais brisée par la perestroïka. La dissolution de l'identité soviétique a entraîné la formation de groupes nationalistes qui pendant quelques années ont défini, soit de façon positive, soit de façon négative, le paysage intellectuel du Kazakhstan ${ }^{42}$. Ainsi, les secteurs les plus élitistes - artistes, écrivains, réalisateurs, qui avaient reçu leur formation entièrement en russe et qui avaient connu une réussite considérable dans l'univers culturel soviétique, comme Oljas Sulejmenov ou Čingiz Ajtmatov — ont été, avec l'implosion de l'Union et le renouveau des cultures nationales, écartés de la scène. Formés dans le cadre multinational des beaux jours de l'URSS, ces

39. A. Gunašev, «Evrazijskij sojuz, eto naša obščaja sud’ba » (L'Union eurasiatique, c'est notre destin commun), Kazahstanskaja pravda, 16/9/1994, pp. 1-2. (trad. angl. Foreign Broadcast Information Service, USSR-94-107, 3/10/1994).

40. Zbigniew Brzezinski, Le grand échiquier : 1'Amérique et le reste du monde, Paris, Bayard, 1997.

41. Ce phénomène est patent dès 1996, parallèlement à l'accumulation des arriérés de salaire impayés. Voir Alain Giroux, «Kazakhstan », Le Courrier des Pays de 1'Est, 417, mars 1997, pp. 38-45.

42. Chez les Kazakhs, une sensibilité nationaliste a sans aucun doute existé bien avant l'indépendance avec des caractéristiques précises. D'abord, par ses caractères intrinsèques dérivant de l'osmose avec les penseurs russes, elle se rapproche des nationalismes historiques européens. À la différence des autres peuples d'Asie Centrale, les Kazakhs, avant même la « délimitation nationale » des années 20, étaient peu enclins à fonder leur identité nationale sur leur appartenance à l'islam; ils se référaient à l'enracinement ethnique, à l'affinité culturelle, au sentiment de commune appartenance historique à une même communauté partageant le même destin. K. H. Karpat, op. cit., p. 313. 
individus - souvent exclus des mécanismes profonds de la culture locale, devenue soudainement nationale - raisonnent par ailleurs en dehors de tout cadre conceptuel fondé sur l'appartenance ethnique ou les liens traditionnels ${ }^{43}$. Ils se sont retrouvés citoyens d'un espace partiellement étranger, perçu comme une île aux contours flous, flottant entre différentes civilisations ${ }^{44}$.

Mais les appels à l'Eurasie n'ont pas seulement signifié la conservation des liens culturels qui reliaient les écrivains dans l'ancien espace de l'Union. En exploitant les nouvelles possibilités médiatiques de la conjoncture postérieure à l'indépendance, la nouvelle intelligentsia nationaliste a éléboré une représentation du réel où tous les problèmes et les questions conflictuelles de la république s'expliquent par la présence d'un intrus, ennemi incarné par les Russes, les Russophones, surtout quand ces derniers sont Kazakhs et ont tous les signes extérieurs de la culture russe. Ainsi le radicalisme des nationalistes allait non seulement impliquer la rupture entre les deux groupes ethniques principaux, mais il allait créer une fracture entre les Kazakhs nationalistes et les Kazakhs « russophones ». La logique même du normativisme nationaliste a imposé à tout intelligent le choix de se ranger soit dans le camp nationaliste soit dans celui des nouveaux «ennemis du peuple ». En ce sens la référence eurasiste s'est appliquée tout d'abord à l'opposition. En fait la question de la langue a été le terrain où s'est consommée l'entente entre intellectuels nationalistes et État, dans le but de faire du critère de la langue le seul valable dans la définition de la nation ${ }^{45}$.

L'âpreté de cette confrontation intestine a incité les intellectuels qui sont restés en dehors de la vague nationaliste et de l'appui de l'État, à recourir à la référence à l'Eurasie pour souligner l'entité de la fracture inévitable sur le plan social entre Slaves et Kazakhs et critiquer le « nationalisme sauvage » émergent. Ils ont qualifié d' « ethnocratique » et d' « apartheid » le nouveau régime ${ }^{46}$, accusé de se servir d'une minorité bruyante de « maîtres de village » ${ }^{47}$ politisés et « ethno-nationalistes » qui serait l'expression d'un pouvoir éloigné des vœux de la majorité de la population ${ }^{48}$.

43. A. Malašenko, « Novye orientacii central'noi Azii i Rossija » (La Russie et les nouvelles orientations de l'Asie Centrale), in Etničeskie i regional'nie konflikty $v$ Evrazii (Les conflits ethniques et régionaux en Eurasie), Moscou, « Ves’ mir », 1997, 3, pp. 207-225.

44. Remarquons que cette perte de statut a assimilé ces personnages aux Eurasistes des années 20 , restés sans patrie et vivant sous la menace constante de l'obligation d'abandonner leur lieu d'origine, dans la même condition d'émigrés et le même état psychologique de crise et de contrainte. Vladimir Gundarev, rédacteur en chef de la revue Niva, Astana, 20/8/1992.

45. A. Kajdarov, « $40 \%$ - eto ne ih vina, a beda » (40\%, ce n'est pas de leur faute, mais c'est un malheur), Kazahstanskaja pravda, 20/8/1992.

46. N. Masanov, N. Amrekulov, «O podlinnom patriotizme i dikom nacionalizme » (Patriotisme authentique et nationalisme sauvage), Karavan, Almaty, 14/1/1994.

47. M. Kirimli, «Emerging nationalism or the new strategy of independent Kazakhstan », Eurasian Studies, II, 2, Ankara, 1995, pp. 2-13. Ces nouveaux acteurs de la politique intérieure constituaient, avant la perestroïka, la base de l'enseignement et étaient complètement en dehors de la nomenklatura et des réseaux culturels soviétiques à travers lesquels l'homme de culture pouvait établir un contact ténu avec le monde extérieur.

48. A. C. Panarin, « Predely faustovskoj kul'tury i puti rossijskoj civilizacii » (Les limites de la culture faustienne et les voies de la civilisation russe), Civilizacii i kul'tury, 3, 1996, pp. 29-55. 
En fait ils ont emprunté une voie essayant de dépasser à la fois les chimères à base raciale et ethnique de l'intégration turcophone proposée par les nationalistes et les chimères idéologiques et passéistes des partis marxistes dans le but de donner une nouvelle forme à l'attraction inévitable entre une métropole et une ex-colonie enserrées dans un contexte continental.

Dans un panorama médiatique subdivisé en deux sphères très décalées selon la langue utilisée, les intellectuels non nationalistes ont été ceux qui ont le mieux compris les problèmes du pays et les orientations qui animaient son opinion publique ${ }^{49}$. Ainsi la référence kazakhe à l'eurasisme a été vite remarquée par les observateurs internationaux.

\section{La société kazakhe et l'eurasisme}

Au-delà des luttes entre intellectuels, l'influence de l'idée eurasiste au Kazakhstan s'explique par l'apparition de fractures internes dans la société kazakhe. On a déjà remarqué comment la distorsion entre la volonté nationaliste revancharde face aux Russes, à la Russie, et l'impossibilité de se passer des cadres, du partenariat et plus particulièrement des références psychosociales émanant du monde russe, ébranlait la société50. Mais plus encore, les nationalistes, à la recherche de liens pour consolider cette société cassée par la perte du soviétisme et l'apparition de rivalités tribales, ont essayé d'instaurer des frontières face à celui avec qui ils ont en réalité beaucoup d'affinités, en exaltant toutes les valeurs particulières des Kazakhs et, d'abord, en utilisant l'islam comme facteur idéologique et politique. Si, pour une partie de la population kazakhe, la pratique religieuse est en progression, en revanche les couches urbaines refusent de s'identifier aux musulmans du monde arabe et craignent au contraire d'être assimilés à cette aire culturelle ${ }^{51}$. De même, si l'on observe le retour de la différenciation des rôles entre les sexes, les femmes kazakhes, qui ont eu accès aux études et au travail pendant la période soviétique et qui ont de ce fait adopté massivement les habitudes vestimentaires et sociales des Russes, constituent une audience potentielle pour l'eurasisme. Ainsi, le retour à l'islam, comme moyen d'affirmation identitaire face à l'autre, contribue au processus de morcellement intérieur du groupe kazakh et renforce le discours eurasiste.

Une autre fracture accentuée par le fait nationaliste est le retour de la légitimité politique de la séparation tribale des Kazakhs, traditionnellement divisés en trois hordes (žuz), la Grande horde (Ulu žuz), la Moyenne horde (Orta žuz) et la Petite

49. B Dave, «Cracks emerge in Kazakhstan's governement monopoly », Transition, 6/10/ 1995.

50. Catherine Poujol, « Minorités exogènes ou Russes de l'intérieur en Asie Centrale », Revue d'Études comparatives Est-Ouest, 1995, pp. 125-142.

51. B. Dave, « Inventing Islam - and an Islamic threat - in Kazakhstan », Transition, 29/12/ 1995, pp. 22-25. 
horde (Kiši žuz) ${ }^{52}$. Depuis 1991, ce fait tribal s'est constamment renforcé. Il est caractérisé par la prééminence politique et économique de la Grande horde sur la Moyenne horde, vivier traditionnel de cadres culturels ${ }^{53}$. La Grande horde n'a pas cessé d'exercer sa mainmise sur le pays, essayant de s'octroyer la majorité des cadres qualifiés ${ }^{54}$. Cette confrontation, qui s'exprime à plusieurs niveaux - le transfert de la capitale vers le nord relèverait d'une même stratégie pour affaiblir l'emprise territoriale de la Moyenne horde sur son espace ancestral - prend parfois des couleurs civilisationnelles, puisque les membres de la Grande horde, au sein de laquelle la renaissance religieuse est la plus forte, ont, dans les régions septentrionales du Kazakhstan, la réputation d'être trop musulmans et trop conservateurs contribuant ainsi au décalage géopolitique du pays ${ }^{55}$. On comprend pourquoi ce clivage fait de la Moyenne horde, ou au moins de ses intellectuels, un pôle distinct du discours eurasiste; on remarquera que $\mathrm{O}$. Sulejmenov, premier Kazakh contemporain à se rapprocher du discours eurasiste, est la figure la plus éminente de ce groupe. Ce pôle se distingue à la fois par les prétentions excessives de l'élite au pouvoir et par les liens privilégiés qu'il essaie d'entretenir avec la Russie. Expulsé des structures nationales, en contact direct avec la frontière «transparente » qui le sépare de la Russie, ce groupe tribal est favorable à la création d'une intégration transfrontalière avec le nord, principalement dans le domaine de l'éducation. La question se pose, sur un plan strictement matériel, par la demande de la levée des obstacles douaniers et autres restrictions afin de faciliter la circulation des deux côtés de la frontière ${ }^{56}$.

La vaste communauté russe du Kazakhstan - pour laquelle le Kazakhstan en tant qu'État national demeure quelque chose d'extérieur, la patrie restant avant tout l'URSS ou le « lieu de naissance \} ^ { 5 7 } \text { — est forcément un pôle fondamental de l'eura- } sisme. En général, les Russes ont une connaissance très vague des contenus positifs de la vision eurasiste qui, pour eux, n'est qu'un moyen de maintenir une certaine forme d'union avec Moscou. Bien qu'il y ait au Kazakhstan des Russes qui choisis-

52. Elles-mêmes subdivisées en tribus puis en clans. N. Masanov, « Kazahskaja političeskaja i intellektual'naja elita : klanovaja prinadležnost' i vnutrietničeskoe soperničestvo » (L'élite politique et intellectuelle du Kazakhstan : appartenance clanique et rivalité inter-ethnique), Vestnik Evrazii, 3, 1996,pp. 46-61.

53. D. A. Mitina, «Migracionnye progressy i tendencii etnosocial'nogo razvitija v Kazahstane » (Processus migratoires et tendances du développement ethno-social au Kazakhstan), in Etničeskij faktor v sovremennom sotsial'no-političeskom razvitii Kazahstana (Le facteur ethnique dans le développement socio-politique actuel du Kazakhstan), Moscou, Rossijskaja Akademija Nauk, 1996 (Institut d'ethnologie et d'anthropologie, document 94).

54. Ainsi à Almaty où se concentrent $70 \%$ des professeurs agrégés (doktor) et $60 \%$ des candidats au doctorat (kandidat), contre $1 \%$ dans le nord en 1994. V. V. Pazurbaev, « Rossijskaja Federacija i 'bližnee zarubez'e'. Geopolitičeskie problemy » (La Fédération de Russie et l' «étranger proche». Problèmes géopolitiques), in Rossija $i$ ee sosedi. Etnonacional'nye otnošenija v novom geopolitičeskom prostranstve (La Russie et ses voisins. Rapports ethniques et nationaux dans le nouvel espace géopolitique), Moscou, 1994, p. 91.

55. N. Masanov, K Kazahskaja političeskaja i intellektual’naja elita .. », art. cit.

56. Mihail Ustjugov, journaliste, entretien téléphonique, Almaty, 2/8/1998.

57. Centre de monitoring des relations inter-ethniques au Kazakhstan, Etnopolitičeskij monitoring $v$ Kazahstane, 2, hiver 1995/1996 (Fonds international Arkor, Almaty, 1996). 
sent de s'identifier à une Eurasie conforme aux termes officiels, pour la majorité d'entre eux, la civilisation eurasiste devient surtout synonyme de russe. Parmi les Russes aussi, il existe une subdivision entre les « purs intellectuels », très proches de leurs collègues kazakhs, et une aile comparable en fait à un nationalisme ethnique. Les premiers reconnaissent une acculturation réciproque avec les nationaux, car, audelà de l'européanisation, il s'est opérée une inversion des caractères culturels qui a rendu les Russes du Kazakhstan plus ouverts et mieux disposés envers les Kazakhs que les Russes «métropolitains ${ }^{58}$. Les autres ont tendance à graviter autour des composants plus chauvins du spectre politique de la Fédération russe. Tous affirment l'existence d'une fraternité avec les nationaux de couches populaires, à laquelle s'opposent une poignée d' intelligenti kazakhs qui trahissent leur peuple en lui proposant de dangereuses chimères vidées de sens ${ }^{59}$. Notre enquête a relevé un grand manque de confiance dans l'avenir, dû à l'insouciance de Moscou ainsi qu'une méfiance à l'égard du projet politique de Nazarbaev, vu comme l'expression d'une ruse orientale, d'une duplicité subtile, utilisée dans un jeu d'oppositions politiques dont le décalage entre idée et réalité « ethnocratique » est dénoncé.

Un dernier pôle est formé des Allemands, pour lesquels l'eurasisme est la seule voie à suivre pour l'avenir du pays, elle ne sera possible qu'au travers d'une articulation organique à l'Europe. Aussi mettent-ils beaucoup d'espoir dans l'unification européenne pour accélérer l'amélioration de la situation au Kazakhstan ${ }^{60}$. Le projet de Nazarbaev avait été perçu à l'origine comme l'expression de cet impératif. L'évolution des événements et l'absence de concrétisation ont engendré une grande désillusion parmi cette communauté. Actuellement, la méfiance prévaut envers les initiatives du pouvoir, même si on s'accorde à rendre la Russie en grande partie responsable de l'impasse dans laquelle se trouve le pays.

\section{L'eurasisme de la haute intelligentsia autochtone}

Le deuxième axe de l'eurasisme, qui prend appui sur la philosophie de l'histoire, s'est développé au Kazakhstan, parallèlement aux images émanant du gouvernement, et souvent en contradiction avec elles. Les secteurs les plus élitistes de l'intelligentsia ont essayé de surmonter l'étroite vision nationaliste et la perte des repères culturels engendrées par l'occidentalisation à travers l'eurasisme. On a vu ainsi surgir une conception dans laquelle la perspective eurasiste devient une idéologie d'intégration, une sorte de néo-humanisme qui - dans la vision de Murat Aouezov, figure importante du panorama culturel et politique, «sanctifié » du fait qu'il est le fils du principal maître-à-penser kazakh du siècle, Muhtar Aouezov —,

58. Entretien avec $\mathrm{Ol}^{\prime}$ ga V. Sarmanova, présidente de l'Association kazakhe des enseignants des écoles russes, Almaty, le 30/7/1998.

59. Entretien avec Jurij Z. Bunakov, leader de la Russkoe Obščestvo, membre du Congrès des peuples du Kazakhstan, Almaty, 3/8/1998.

60. Entretien avec A. Dederer, président de la Communauté allemande du Kazakhstan, Almaty, 14/8/1998. 
sert de base commune à tous les peuples du Croissant islamique, de la Chine et du Sous-continent indien, et offre une réserve de réponses à toutes les questions qui surgissent dans les contacts entre ces civilisations ${ }^{61}$. Pour donner un caractère objectif à cette version de l'eurasisme, ses acteurs ont recours à un arrière-fond historiosophique où l'espace eurasien est conçu comme axe de l'histoire universelle. L'Eurasie se définit en particulier par un « âge des prophètes », dont la véritable essence réside dans le fait d'être la source des religions mondiales ${ }^{62}$.

Le point à noter dans cette interprétation est l'ouverture «humaniste » par laquelle ces intellectuels kazakhs se démarquent de leurs collègues de Moscou et de Saint-Pétersbourg. Ces derniers essayent à travers l'eurasisme de délimiter un espace commun entre les Slaves orientaux et les peuples turcophones continentaux ce qui exclut des rapports étroits avec le monde occidental, l'Islam et la Chine. La Chine est considérée au contraire comme un pôle constitutif de l'Eurasie ${ }^{63}$. La version russe est donc accusée de diminuer l'importance des liens que les peuples de la steppe avaient renoués avec ces civilisations, pendant le Moyen Age et même avant. La compréhension de l'Eurasie impose donc de sortir d'une psychologie collective prisonnière du passé, et d'envisager des directions de rencontres interculturelles qui dépassent le camp post-soviétique pour s'étendre à un « sub-continent eurasien » comprenant tous les peuples du vieux continent ${ }^{64}$.

En dégageant une tendance mondiale à l'intégration, ce discours nie le sens même de l'eurasisme en tant que projet politique, ce qui était aussi une des composantes principales de la version russe. Il faut rester loin des formules politiques concrètes puisque tout modèle systématique ne peut être qu'une déformation de l'héritage spirituel de l'Eurasie, fondamentalement universel. L'eurasisme est structurellement dans l'impossibilité d'établir des modèles contemporains de construction étatique ou de servir de base à l'intégration entre les peuples post-soviétiques. M. Aouezov tranche net en déclarant que le projet de Nazarbaev, qui se tient en dehors de la réalité historique et de sa connaissance, est conjoncturel et donc condamné à un rapide oubli.

\section{L'eurasisme d'État au Kazakhstan}

Cet eurasisme flou, qu'on a vu émerger comme une tentative de réponse aux difficultés de la société et comme l'expression d'un parti, le Congrès du peuple du

61. Les affirmations sur cet aspect de la sensibilité eurasiste au Kazakhstan se fondent principalement sur des entretiens personnels avec O. Sulejmenov et M. Aouezov, principales figures intellectuelles du Kazakhstan contemporain.

62. Elle est vue comme le théâtre, au milieu du premier millénaire avant notre ère, de l'accouchement de la pensée de Confucius et Lao Tseu en Chine, de la confrontation entre bouddhisme et hindouisme en Inde, du zoroastrisme en Perse jusqu'à un changement du rôle du mythe en Grèce. Entretien personnel avec Murat Aouezov, Almaty, 21/8/1998.

63. O. Sulejmenov, entretien cité.

64. M. Aouezov, entretien, « Glavnoe ne molčat` » (Surtout ne pas se taire), Karavan, 10/4/1996. 
Kazakhstan, et de la sensibilité de son président, Oljas Sulejmenov, a été ensuite instrumentalisé par l'État. Cette présence de l'État en tant que premier acteur est un fait majeur du discours eurasiste au Kazakhstan, ce qui le place dans une position tout à fait différente du reste de l'espace post-soviétique. Bien que souvent très rhétorique et peu conséquent, ce volet institutionnel du discours eurasiste demeure d'un grand intérêt. Son but est de fournir des repères utiles à la construction d'une nouvelle identité, étatique aussi bien que sociale.

\section{L'eurasisme dans les stratégies d'intégration des ethnies : du « kazakh » au « kazakhstanais » à l' « eurasiatique »}

Bien évidement, la nécessité de maîtriser une hétérogénéité ethnique extrême continue à alimenter le discours eurasiste au Kazakhstan. Ici, plus que dans les autres NEI, on perçoit toute la perversité du mécanisme soviétique de la «nationalité titulaire» et de sa scission entre citoyenneté «civique» et « ethnique », fait qui, après 1991, plaça des millions d'individus dans une position juridique incertaine. Il était donc impératif de donner des repères identitaires communs à une société ethniquement éclatée. Nazarbaev a senti que les idées eurasistes dans le cadre multinational du Kazakhstan étaient tout à fait cohérentes.

Cette utilisation est le résultat d'une évolution des stratégies du pouvoir. Initialement, pour neutraliser l'opposition des forces nationalistes, les seules potentiellement en mesure de défier sa légitimité entre 1989 et 1993, le pouvoir s'est livré à l'exploitation de ses thèmes, en promouvant l'idée ethnique kazakhe comme base de l'unité du pays. Ceci a engendré une politique ouverte de kazakhisation généralisée, au cours de laquelle on a essayé d'imposer la prédominance des Kazakhs de souche dans l'administration. Cette démarche a été rapidement qualifiée, par des observateurs internes au Kazakhstan, d'ethnocratique et relevant de l'apartheid 65 .

Cette stratégie fut source de lourdes distorsions à la fois sur le plan intérieur pour les raisons analysées ci-dessus et sur le plan international car les efforts tendant à favoriser le retour des Kazakhs de Mongolie et de Chine ont eu une action immédiate sur les délicats équilibres ethniques du Xinjiang ${ }^{66}$. La phase ethnocratique a été entièrement faussée dans ses prémisses mêmes, fondées sur un triomphalisme démographique qui prévoyait un changement des équilibres internes dans les années à venir en vertu des seuls courants natalistes des deux ethnies principales. Mais le désastre économique a fait échouer la revanche des berceaux kazakhs en

65. N. Masanov, «Nacional'no-gosudarstvennoe stroitel'stvo v Kazahstane: analiz i prognoz » (L'édification de la nation et de l'État au Kazakhstan : analyse et prospective), Vestnik Evrazii, 1, 1995,pp. 117-128.

66. Ce mouvement migratoire, après avoir connu une flambée initiale, ne concerne plus, à partir de 1995, que quelques centaines de personnes. G. Mukanova, «Migracii Kazakov v Central'noj Azii : vzgljad v prošloe i popytka prognozirovanija » (Les migrations des Kazakhs en Asie Centrale : regard sur le passé et essai de prospective), Vestnik Evrazii, 2 (3), 1996, pp. 64-73. 
raison de l'accroissement de la mortalité infantile dans les campagnes en voie de tiers-mondialisation et de la stabilisation du flux des migrants russes.

Pour pallier les dangereux dérapages de l'ethnocentrisme, le pouvoir a essayé d'introduire le concept d'une identité supranationale « kazakhstanaise ${ }^{67}$, entendant ainsi forger l'identité de tous les ressortissants de la république d'après une idée non ethnique de la nation, sans pour autant négliger le rôle de la nation kazakhe, ethnie d'État dont la renaissance ne doit pas empêcher le développement des autres peuples. Or l'introduction de références eurasistes semble signer une troisième phase de ces manipulations identitaires. Si le sens principal de l'introduction de la référence «kazakhstanaise » résidait dans un concept de citoyenneté commune, distinct de celui d'une nouvelle communauté ethnique, ce pas semble avoir été franchi avec l'utilisation de la perspective eurasiste définie comme «vision du monde qui, en utilisant les résultats de plusieurs sciences, étudie et explique les régularités du développement des différents peuples, comme participants d'un seul processus ethnogénétique de la biosphère de la planète »68. Ainsi, dans ce nouveau cadre, le peuple du Kazakhstan, bien que hétérogène par sa composition ethnique, est présenté en tant que « sujet unique et indissoluble des relations constitutionnelles » et en tant que «communauté de personnes unies par la destinée ${ }^{69}$. En ce sens le retour aux références collectives soviétiques est encore plus accentué - on parle en fait de « rapprochement ( sbliženie) des consciences nationales »70-, mais ce qui est mis en avant ici ce n'est pas l'idéologie, ce sont l'espace et le passé commun de la « nation kazakhstanaise ». Ainsi, le peuple du Kazakhstan serait le résultat de l'adaptation humaine aux conditions physiques de l'Asie intérieure, par un processus d'acculturation mutuelle accouchant d'un peuple de mentalité différente. Selon le professeur V. Injušin, on serait ainsi en présence d'un ethnos «synthétique », puisque les Russes présents au Kazakhstan depuis plusieurs générations seraient porteurs d'une mentalité particulière et proche de celle des « titulaires ${ }^{71}$.

\section{L'appareil de propagande : un État « idéocratique »}

Le gouvernement a misé constamment sur l'ambiguïté puisque, en même temps, par le biais de la promotion de la langue et de la culture kazakhes, il suscite bien l'identi-

67. Concept inséré dans la nouvelle constitution de 1995 qui débute par le slogan « Nous, le peuple du Kazakhstan », et repris par le président qui se déclare être le président « de tous les Kazakhstanais », quelles que soient leur nationalité et leur religion. Nesavisimaja gazeta, 15/ $11 / 1995$.

68. Il serait de surcroît une méthode interdisciplinaire fondée sur l'utilisation des données de l'archéologie, de l'anthropologie, de la géologie et de l'ethnographie. Evrazija kontinent soglasija - Populjarnye zametki ob integracii etnosov (L'Eurasie, continent de l'entente Notes pour le public à propos de l'intégration des ethnos), s.1., Znanie, 1998, p. 26.

69. Ibid.

70. Ibid.

71. V. N. Hljupin, « Ideokratja prezidenta Nazarbaeva : idei, instituty, elity » (L'idéocratie du président Nazarbaev : les idées, les institutions, les élites), Voshod, 3, 1997, pp. 128-136. 
fication entre nation et groupe kazakhs ${ }^{72}$. Cette ambiguïté semble relever d'une stratégie complexe induite par la conjoncture multi-ethnique du Kazakhstan, dont le but est sûrement de créer un climat de confiance au sein de la communauté russe et d'éviter l'utilisation de la catégorie de « russophones », très politiquement incorrecte pour le nouveau régime puisque susceptible d'augmenter le front de son opposition ${ }^{73}$.

Dans la comparaison avec la Russie, le rôle et la nature de l'État constituent un point fondamental du débat. Si, en Russie, le souci initial de démocratisation menait à une remise en cause radicale d'une tradition incontestablement solide, qui s'apparentait parfois à la honte, à l'opposé, au Kazakhstan, le problème était de s'attacher à tous les repères historiques justifiant l'existence d'une tradition étatique nomade pré-soviétique. Ainsi on peut entendre parler d'une «nation formée par l'État» (gosudarstvennoobrazujuščaja nacija) ${ }^{74}$. Or, un point fondamental du discours eurasiste, basé sur l'étude de Gumilev à propos de l'étatisme des steppes, consistait à considérer l'État comme lieu réel de l'identification d'individus, agents à leur tour de l'expansion de son modèle culturel. Dans toutes ces références, on remarquera l'appropriation par l'État kazakh de la figure de Gumilev, essentielle dans la réception de la vision eurasiste kazakhe. Ses livres - réévaluant le rôle de l'Asie dans l'histoire russe et octroyant aux peuples et aux Empires de la steppe une haute dignité culturelle - ont été rapidement épuisés au Kazakhstan dés le début de la décennie. Dans la tâche difficile de leur recherche identitaire, les dirigeants ont utilisé le concept de mestorazvitie - qui pourrait se traduire en français par « développement en conformité du lieu » - déjà présent chez les premiers Eurasistes, mais largement développé dans les travaux de Gumilev ${ }^{75}$. En outre l'œuvre de ce dernier est considérée comme affranchie de la russophilie, qui, surtout aux yeux des non-Russes, pesait sur les travaux des Eurasistes des années 2076. Cela serait dû au fait que Gumilev a vécu dans la république, et qu'il a consacré son œuvre aux peuples turcophones. De manière générale, le savant russe jouit d'une réputation inattaquable dans les milieux universitaires du pays comme expert des peuples turciques de la steppe. Il est considéré par certains comme l' « Einstein de la recherche sur la steppe ${ }^{77}$, et son nom a été donné à l'université de la nouvelle capitale $^{78}$. Ici et dans la majorité des établissements supérieurs du pays, l’Eurasie

72. I. Ohayon, Renouveau culturel et identitaire au Kazakhstan, 1989-1996. Approche politique et sociale, Paris, INALCO, mémoire de maîtrise, 1997.

73. Pal Kolstø, « Anticipating demographic superiority : Kazakh thinking on integration and nation building », Europe-Asia Studies, 50, 1, 1998, pp. 51-69.

74. G. Tanirbergernova, « Regional’nyj opyt realisacii vnutrennei politiki » (Expérience régionale de réalisation de la politique intérieure), Sajasat, 5, 1995.

75. L. Gumilev, Etnogenez i biosfera zemli (Ethnogénèse et biosphère de la terre), Leningrad, Gidrometeoizdat, 1990,p. 185.

76. E. Saudabekoga, «Evrazijstvo Gumileva i klassičeskoe russkoe Evrazijstvo » (L'Eurasisme de Gumilev et l'Eurasisme russe classique), Mysl', 8, 1997, pp. 30-34.

77. Entretien avec l'archéologue V. F. Zaibert, Almaty, 6/8/1998.

78. Sur les quelque cinquante personnalités de pointe du monde culturel et universitaire kazakh interviewées, toutes n'ont eu que des mots enthousiastes pour définir le savant russe. 
est devenue le sujet de prédilection des thèses de doctorat, comme l'était auparavant l'histoire du PCUS. Toutefois, en dépit de la notoriété actuelle de son auteur, il semble que l'œuvre de Gumilev, en raison de la difficulté des chercheurs contemporains à en étudier la richesse et la complexité, ne soit réellement connue que de cercles restreints.

En fait ce qui est important ici c'est que l'État opère un processus d'appropriation du prestige de la culture russe comme élément de l'héritage du Kazakhstan.

Pour comprendre ces manipulations, il faut enfin les placer dans le contexte d'un État de moins en moins démocratique et devenu la « chose » privée du président. À côté de l'insertion des critères tribaux et claniques, l'autocratisation présidentielle a entraîné le contrôle total des médias et leur orientation vers le détournement du passé et la construction de toutes sortes de représentations identitaires. Pour répondre à ses objectifs autocratiques et grâce à des moyens de financement considérables, le président Nazarbaev a donc mis en place un appareil ad hoc pour contrôler les médias, contribuant ainsi au maintien des procédés soviétiques ${ }^{79}$. Ce qu'on a vu surgir est en effet une véritable industrie culturelle, productrice de représentations visant à la diffusion des doctrines officielles de Nazarbaev, où toutes les forces intellectuelles dont il s'est assuré le concours ont été mobilisées ${ }^{80}$. L'industrie culturelle étatique apparaît comme une structure pyramidale organisée autour de centres principaux relevant directement de la présidence et expression de l'élite de la Grande horde ${ }^{81}$. Parallèlement, des centres d'analyse se multiplient exprimant des visions différentes, parfois concurrentes des organismes cités, fondées sur un nationalisme plus fort et faisant référence à la Petite ou à la Moyenne horde. Cette emprise du pouvoir sur la production culturelle s'est dotée de Centres de gestion des rapports avec la presse parmi lesquels vient en tête un Service de presse du président, où l'on trouve un groupe important d'image-makers et un « Centre de technologies politologiques ». Les conceptions officielles sont en outre développées au cours de conférences et autres actions médiatiques organisées en vue de promouvoir l'image du président.

La prolifération des centres d'analyse et de production idéologique témoigne de la permanence de dispositions psychologiques fortement enracinées. En ce sens, les slogans « Kazakhstan $2030 »$ ne sont que la prolongation des campagnes promettant de «magnifiques destinées progressistes » au peuple.

79. L’information est enfin largement structurée par les émissions de la Fédération russe. La situation des médias met en évidence la corrélation entre leur jouissance et les attitudes des lecteurs, fait qui structure un tableau général d'aliénation de la majorité de la politique du pays. Une enquête de Radio Free Europe/Radio Liberty dresse le même constat. Cf. M. Haney, « Alienation in Almaty : Ethnicity and information », Transition, 28/4/1995, pp. 19-22.

80. V. N. Hljupin, op. cit.

81. On mentionnera aussi l'Institut du développement du Kazakhstan, éditeur des revues Saljat - Politika et Evrazijskoe soobščestvo. Le volet international de l'image du pays a été directement élaboré par le Centre d'information et d'analyse de l'appareil présidentiel (IAC), dans lequel la femme du président tient un rôle important. 


\section{L'eurasisme d'État, son utilisation dans la politique extérieure}

La référence eurasiste au Kazakhstan comporte également un projet d'Union Eurasiatique d'États (UEA, Evraziskij Sojuz Gosudarstv), lancé par Nazarbaev en 1994.

Vue sous cet angle, la question eurasiste quitte le domaine de la recherche identitaire et intellectuelle exprimée par le terme « civilisation », et rejoint la problématique, bien connue des Européens, de la recomposition des espaces régionaux. Le président lui-même a tenu à souligner que le projet émanait non seulement de réalités historiques et culturelles mais encore de facteurs strictement pragmatiques. Abandonnant certains des propos isolationnistes de la période d'après 1991 et reconnaissant la fatalité d'une attraction réciproque, les Nouveaux États Indépendants n'ont trouvé dans la CEI presque aucune des conditions de cette coopération. Devant une CEI inopérante, on a vu naître une série de mouvements d'intégration dans pratiquement toutes les régions autour de l'ancienne Union - réactivation de l'ECO, création de la Zone économique de la mer Noire, renforcement de l'Union européenne, nouvelles géométries dans le Pacifique - qui ont laissé les NEI dans une position incertaine ${ }^{82}$. Le discours sur le maintien et la rénovation d'un cadre cohérent d'intégration figure parmi les questions où le concept d'Eurasie est le plus utilisé83. Redoutant les risques d'isolement mondial, il souligne l'échec des programmes de transition indépendants et notamment l'impossibilité de réorienter le système économique propre au Kazakhstan vers les organisations économiques régionales extérieures. Dans la politique étrangère de l'État kazakh, le projet prend une connotation d'intégration régionale faisant pendant à l'Union européenne, afin d'insérer le pays dans une recomposition des relations internationales à l'heure de la globalisation ${ }^{84}$.

Le projet s'insère avec cohérence dans la biographie politique du président kazakh, infatigable soutien de toutes les initiatives de Gorbačev au cours de l'année 1991 pour la recomposition d'une Union d'État souveraine, et qui s'est vu offir le poste de premier ministre par Gorbačev et par El'cin ${ }^{85}$.

Si donc quelques jours encore avant la dissolution de l'URSS, Nazarbaev déclarait que « personne n'arriverait jamais à arracher du cœur de notre peuple le sentiment puissant d'appartenir à une Patrie unique (soviétique) », 86 il serait intéressant de savoir pourquoi, à un moment donné, il lui a apposé l'étiquette d' «Eurasie ». Il avait probablement entendu le mot prononcé par Andrej Saharov qui, dans les

82. A. Voskressenkij in M. M. Tazin, ed., Evrazijskoe prostranstvo..., op. cit., p. 100

83. Voir par exemple A. Bykov, S. Dyza, «Problemy formirovanija evrazijskogo ekonomičeskogo prostranstva » (Les problèmes de la formation d'un espace économique eurasiatique), Vnešnjaja torgovlja, 4, 1997, pp. 38-42.

84. W. Dressler, La reconstruction des rapports russo-kazakhs depuis 1991 ou les aléas de l'utopie eurasiatique, Paris, Centre français du commerce extérieur, mai 1998.

85. Entretien avec Mihail Gorbačev, « Meždu Ljuterom i papoj » (Entre Luther et le pape), Karavan, 19/9/1997.

86. N. A. Nazarbaev, Sans droites ni gauches, Lausanne, L'Âge d'homme, 1993. 
discussions sur la réforme de l'Union, avait parlé d'une «Union des Républiques Euro-asiatiques » (Sojuz Evro-Aziatskih Respublik). Puis, en 1992 au Kazakhstan, le programme du parti de Sulejmenov soutint un projet de traité confédéral sur le modèle européen, lui aussi nommé evrazijskij.

C'est alors que le président a utilisé ce nouveau terme en l'appliquant à son initiative de renouveau de l'Union, évidemment tenté par le mélange subtil de culture et de pouvoir auquel la formule l'autorisait ${ }^{87}$. Parmi les personnalités qui ont défini cette ligne il faut citer l'écrivain Abiš Kekilbaev, inspirateur supposé de bon nombre des idées de Nazarbaev ${ }^{88}$.

Au cours d'une visite officielle à Moscou (en mars 1994), le président a exprimé la nécessité d'une forme d'intégration des économies et des systèmes éducatifs et de sécurité post-soviétiques, sur les bases nouvelles de l'économie de marché 89. Union de « sujets égaux et indépendants », l'UEA fut présentée comme une organisation multilatérale et intégrée, potentiellement de type confédéral dont le modèle était, bien entendu, l'acquis communautaire européen.

Le projet eut une forte résonance médiatique qui stimula en effet le débat sur la nécessité d'une révision de l'intégration. Dans les accords successifs entre Russie, Biélorussie, Kazakhstan et Kirghizie, des échos de cette proposition ${ }^{90}$ offraient à la presse l'occasion de parler de la formation d'une nouvelle «Union des États eurasiatiques » avec transfert de souveraineté. Ces commentaires enthousiastes n'ont pas trouvé de correspondances dans les déclarations des présidents, très modérées, qui évitaient de s'exprimer sur la portée des articles de l'accord. La question principale était liée à l'utilisation du terme sojuz : celui-ci exprime une union au sens fédéral, alors que les textes signés ne prévoyaient pas les modalités de l'exécution des normes. C'est donc un malentendu sémantique évident qui dérive de l'utilisation de la terminologie occidentale. Dans les faits, au-delà des réactions favorables à l'intégration eurasiatique, à l'intérieur de la Fédération de Russie, les critiques d'un projet dont le système de vote aurait mis trop facilement en minorité le membre le plus important de l'Union furent très nombreuses. Une grande méfiance a été enregistrée en dehors de l'axe Moscou-Astana.

On peut s'interroger sur les raisons du projet alors que rien d'effectif n'a suivi. D'abord, il ne faut pas oublier que 1994 est l'année du « retour de l'espace dans la

87. O. Sulejmenov, entretien cité.

88. V. Gunderev, entretien cité.

89. N. A. Nazarbaev, «Evrazijskij Sojuz Gosudarstv. Proekt o formirovanii evrazijskogo sojuza gosudarstv. » (L'Union eurasiatique d'États, 3/6/1994. Projet pour la formation d'une Union eurasiatique d'États, 3/6/1994) in Kazahstansko-rossijskie otnošenia, 1991-1995 gody. Sbornik dokumentov i materialov. Posol'stvo Respubliki Kazahstana v Rossijskoj Federacii (Les relations kazakhstano-russes, 1991-1995. Recueil de documents et de matériaux. Ambassade de la République du Kazakhstan en Fédération de Russie), Almaty - Moscou, 1995, pp. 366-376

90. Texte de l'accord entre Biélorussie, Kazakhstan, Kirghizie et Fédération de Russie sur l'approfondissement de l'intégration dans les secteurs économiques et humanitaires, InvestKur'er, Moscou, juin 1996, pp. 3-14. 
politique de Moscou », ${ }^{91}$ où l'eurasisme apparaît de plus en plus comme l'instrument d'une nouvelle politique russe. El'cin affirme un droit de regard de Moscou sur l'espace post-soviétique, la Russie ne pouvant pas abandonner son « ventre mou » méridional. Il s'agissait d'assouplir ce processus et d'empêcher ainsi qu'il ne prenne des formes trop impérialistes. C'est alors que Nazarbaev agit sur l'aile nostalgique de l'intelligentsia moscovite, en favorisant la publication de la revue Evrazija, narody, kul'tury, religii. Dans la conjoncture politique de 1996, marquée par la dénonciation des accords de Belovežskij par le front social-patriotique, il était fondamental pour les élites au pouvoir que, à la veille des élections, le thème de la nécessaire intégration de l'espace post-soviétique ne restât pas une exclusivité de l'opposition.

Le programme de l' «intégration eurasiatique » a longtemps primé dans le discours officiel de la présidence. Ainsi, le but principal de Nazarbaev sur le plan intérieur était évidemment d'affaiblir la tension ethnique à travers des actions soulignant la volonté de coopération inter-ethnique et interculturelle. Sur le plan extérieur, il s'agissait de renforcer l'image internationale de Nazarbaev comme acteur sincère de la collaboration dans la CEI auprès des audiences extérieures, notamment russes. L'initiative du président a joui en effet d'une très grande couverture médiatique, le projet a été publié intégralement dans de nombreux quotidiens et une multitude d'articles et d'analyses lui ont été consacrés ${ }^{92}$. Les réflexions sur l'eurasisme ont permis d'éviter l'isolement médiatique de la république. Ce fut une manœuvre parfaitement réussie, à en juger par le rayonnement du projet et de l'image publique de Nazarbaev ${ }^{93}$. Par exemple, dans les pages de Nezavisimaja gazeta, le rédacteur en chef, $\mathrm{V}$. Tretiakov, a longtemps décrit Nazarbaev comme le meilleur politicien de la CEI. L'idée a donc atteint son but, même si les louanges ont plutôt laissé la place à la désillusion et si, dans les derniers temps, on peut relever une attitude très négative des médias russes à l'adresse du Kazakhstan. En ce sens le projet est devenu le catalyseur des forces visant à la recomposition. Mais, jusqu'à nos jours, il n'a été suivi d'aucun effet concret ${ }^{94}$.

De nombreux éléments permettent de considérer l'initiative de la présidence kazakhe comme une manœuvre conjoncturelle. Cela semble confirmé par le dernier document idéologique émanant de la présidence, «Kazakhstan - 2030 », où l'eurasisme est présenté comme une des potentialités extérieures, en tant qu'idée élaborée par la présidence afin d'exploiter au mieux la position géographique du pays entre Europe et Asie ${ }^{95}$. Le Kazakhstan de 2030 doit, en effet, devenir le

91. A. C. Panarin, « Predely... », art. cit.

92. On peut signaler en particulier toute une série de tables rondes de haut niveau avec la participation de parlementaires et de chercheurs qui, durant plusieurs jours, se sont interrogés sur le sens et la directions de la collaboration entre «ex-frères ». Notamment la conférence de décembre 1994 à Almaty, avec la participation de plus de 140 dirigeants politiques et sociaux.

93. Sergej Aleksandrovič Panarin, directeur de la revue Vestnik Evrazii, Almaty, 10/8/1998.

94. Nezavisimaja gazeta, 17/2/1996.

95. N. A. Nazarbaev, Kazakhstan - 2030, message du président de la république au peuple kazakhstanais, Almaty, 10/10/1997. 
« centre de l'Eurasie » et le « lien économique et culturel entre trois régions en forte croissance : la Chine, la Russie et le monde musulman ${ }^{96}$. L'eurasisme est ainsi présenté comme un facteur stratégique lié au contexte de la mondialisation économique, avec des résonances de projets sur la «nouvelle route de la soie », en faveur de la coopération « avec les autres pays de la région et avec le soutien des organisations internationales »; le tout dans la plus grande indétermination et sans qu'aucun axe stratégique ne soit défini.

Il faut aussi rappeler l'impact du projet sur les équilibres centrasiatiques. L'intention de Nazarbaev était probablement de contrecarrer les propositions ouzbekes visant à la constitution d'un « Grand Turkestan, maison commune » des peuples de la région ${ }^{97}$, projet qui avait été vite perçu par les autres pays comme une tentative de Tachkent de conserver son hégémonie régionale ${ }^{98}$. Il faut remarquer que le projet d'Union eurasiatique coïncide avec la réouverture du dossier de l'intégration régionale - entre la création de la Communauté (Sodružestvo) des États d'Asie Centrale (Tachkent, 4 janvier 1993) et la naissance de «l'espace économique centrasiatique » (déclaration d'Almaty, juillet 1994) - comme pour assurer ses arrières face à son redoutable voisin méridional. Bien entendu, le projet de Nazarbaev, qui voulait jouer sur deux tableaux, n'a pas rencontré l'accord du président Karimov (aux yeux duquel il est «mort-né ») ${ }^{99}$ et il a été dénoncé dans la presse officielle ouzbeke qui l'a qualifié d'un « retour à l'URSS ».

Cela explique la sclérose ultérieure du projet de l'UEA. En effet, pour le Kazakhstan l'absence de l'Ouzbekistan (et de l'Ukraine) dans la nouvelle union signifierait, outre l'affaiblissement d'un important secteur de ses relations commerciales, une union bilatérale trop déséquilibrée entre la Fédération de Russie et le Kazakhstan qui conduirait à l'absorption inévitable de ce dernier.

\section{Conclusion}

Enveloppe idéologique aux contours flous, issue de la dislocation de l'URSS, l'eurasisme est devenu l'objet d'un débat sur les principaux problèmes que traverse le Kazakhstan. Construction symbolique utilisée par différentes forces politiques avec des objectifs opposés, l'eurasisme au Kazakhstan aide à négocier l'avenir très incertain du pays, tandis qu'au même moment il renforce l'arsenal idéologique d'un pouvoir déconcertant par sa manipulation médiatique des symboles. Il est donc nécessairement flou, l'enveloppe pouvant être remplie par des discours diffé-

96. Ibid., p. 55.

97. I. Karimov, Turkestan, naš ob̌čij dom (Le Turkestan, notre maison commune), Tachkent, Uzbekistan, 1995.

98. Un Turkestan politique demeure un concept purement virtuel et dépourvu d'antécédents historiques car on ne peut pas considérer comme précédents l'administration gouvernementale générale tsariste ou la République soviétique autonome qui ont porté ce nom.

99. Nezavisimaja gazeta, 21/6/1994. 
rents selon le destinataire, l'opportunité du moment et selon l'usage interne ou externe qu'on veut en faire.

Parfois utilisé pour donner aux Russophones l'impression que la république reste dans l'espace de Moscou, le discours eurasiste n'est pas qu'une manœuvre : il exprime la nécessité brûlante d'un idéal pour unir le pays qui sombre dans le sousdéveloppement. À l'intérieur du pays, l'utilisation de la rhétorique eurasiste a sûrement contribué à éviter des conflits ethniques, tout en maintenant un semblant de démocratie. En tant que seul État d'Asie Centrale foncièrement slavo-turcique, le Kazakhstan ne peut pas suivre la politique de mise à l'écart des minorités slaves pratiquée par ses voisins. En ce sens, cette référence reflète la volonté d'intégrer les éléments bénéfiques de l'influence russe qui sont partie essentielle de l'identité européenne revendiquée par l'actuel régime du Kazakhstan face aux dimensions des civilisations asiatiques et musulmanes, qui traversent le pays sans en définir l'essence pour autant.

Souvent présentée comme une couverture destinée à faciliter le divorce d'avec Moscou, la présence du discours eurasiste révèle bien les grandes interrogations sur l'avenir qui attend les deux géants eurasiatiques. Ainsi, le recours du Kazakhstan à l'eurasisme s'explique par le désordre qui a succédé à la partition bipolaire du monde et qui a ébranlé les repères géographiques de cette région centrasiatique revenue au centre des questions stratégiques. Face à la croissance ouzbeke et chinoise, et à la poursuite de la pression russe dans la bataille pour la Caspienne, le Kazakhstan a du mal à devenir un acteur au sens plein. Dans son instabilité, le pays se trouve à mi-chemin entre la Russie et le monde islamique. Pour l'instant il sert de tampon entre les deux; avec le temps il peut évoluer soit vers l'un soit vers l'autre. Dans un tel contexte, l'eurasisme est l'expression de forces troublées par cette incertitude stratégique. En fait, le Kazakhstan doit trouver à travers l'eurasisme sa nouvelle identité régionale tout en gardant une porte ouverte sur la Russie, qui représente à son tour l'ouverture directe sur « l'espace monde »100.

En conclusion, il faut aussi rappeler les évolutions très concrètes qui laissent présager la précarité d'une destinée eurasiste pour le Kazakhstan. Le développement de la vision eurasiste était le reflet du climat socio-politique d'il y a cinq ans, que l'on peut caractériser comme une période de romantisme politique très différente de la réalité d'aujourd'hui qui voit les idées-maîtresses s'effondrer avec la chute de l'autorité morale des leaders politiques ${ }^{101}$. Dans ce vide, la donnée principale chez les Kazakhs reste un attachement aveugle et absolu à la souveraineté, attachement soigneusement entretenu par le pouvoir à travers la définition ambiguë de la nationalité. Le pays poursuit le processus de remplacement de la hiérarchie ethnique soviétique par une hiérarchie kazakhe, ce qui ne fait qu'accroître l'envie d'émigrer et le mécontentement de la majorité des Russes. La création d'un climat de tension inter-ethnique et de marginalisation, sinon d'exclusion, a hypothéqué

100. Jean Radvanyi, ed., De l'URSS à la CEI, 12 États en quête d'identité, Paris, Ellipses, 1997. 101. Vasilij Nikolaevič Vasilenko, expert du KISS pour les problèmes écologiques, fondateur du Congrès du peuple du Kazakhstan, 20/8/1998. 
toute future stratégie d'intégration des groupes ethniques dans le nouvel État, tel l'eurasisme. Sur le plan social, au-delà de toutes les déclarations favorables de l'intelligentsia, une véritable sensibilité eurasiste (si on peut parler en ces termes) parmi les nationaux est l'expression des secteurs les plus marginaux de la société, les « métis » (au moins $5 \%$ ), les citadins de longue date, les russifiés. Et, à l'heure actuelle, personne ne peut encore exclure l'hypothèse de l'entrée du pays dans le croissant islamique. On attend d'observer les effets des réseaux éducatifs turcs sur les nouvelles générations ${ }^{102}$.

Il faut encore prendre en compte certains traits de la culture politique locale. Les Kazakhs idéalisent l'État et ses symboles à travers la personnalité du président ${ }^{103}$, les individus hésitent à s'engager et ce fait a pesé sur les intellectuels, qui s'expriment de moins en moins ou ne font que reprendre les discours présidentiels. Ainsi les données de notre enquête permettent d'observer (chez presque tous les cadres culturels kazakhs interrogés) une croyance diffuse en l'existence d'une civilisation eurasiatique qui se traduit par la répétition quasi mécanique des propres mots du président. Le problème majeur se situe probablement là : le manque profond de connaissance et de débat autour d'une vision du monde qui, d'abord condamnée au silence des catacombes du régime soviétique, a subi lors de sa diffusion par la suite toutes sortes de manipulations. En dépit d'une importante médiatisation, force est de remarquer que le concept d'Eurasie demeure, en dehors de petits cercles, terra incognita pour la majorité des citoyens post-soviétiques. De plus il est certain que l'acculturation croissante à l'Occident empêche une appréhension du véritable sens du terme « Eurasie ».

L'eurasisme va-t-il apparaître comme irrévocablement inutilisable? Le temps va mûrir la réponse. Même si l'idée eurasiste est passée pour l'instant au second plan dans le discours officiel, la diffusion tous azimuts du terme « Eurasie » laisse à penser que cette image pourra être à nouveau évoquée dans l'élaboration des choix politiques. Par ailleurs, le déplacement de la capitale à Astana est une preuve supplémentaire de la persistance de la vocation «eurasiste» du Kazakhstan, surtout dans la continuité d'une crise qui fait paraître l'intégration comme la seule issue possible.

Via Vittorio Veneto, 44

Villadeati $(A L)$

15020 Piémont

Italie

e-mail :fvielmi@tin.it

102. Catherine Poujol, «Est-il facile d'être jeune en Asie Centrale? » in Les pays de la CEI, Paris, La documentation française, 1997, pp. 34-54.

103. Récente confirmation d'un thème classique dans l'enquête du Centre de monitoring des relations inter-ethniques au Kazakhstan, Etnopolitičeskij monitoring..., op. cit. 